\title{
Endogenous Oleoylethanolamide Crystals Loaded Lipid Nanoparticles with Enhanced Hydrophobic Drug Loading Capacity for Efficient Stroke
}

\section{Therapy}

\author{
Shichao $\mathrm{Wu}^{\mathrm{I}-4, *}$ \\ Di Liao ${ }^{3-5, *}$ \\ Xi Li ${ }^{3-5}$ \\ Zeyu Liu $^{3-5}$ \\ Lin Zhang ${ }^{3-5}$ \\ Fong Ming Mo ${ }^{1,2,4}$ \\ Shuo $\mathrm{Hu}^{1,2,4}$ \\ Jian $X_{i a}{ }^{3-5}$ \\ Xiangrui Yang ${ }^{1-4}$ \\ 'Department of Nuclear Medicine (PET \\ Center), Xiangya Hospital, Central South \\ University, Changsha, Hunan, 410008, \\ People's Republic of China; ${ }^{2}$ Key Laboratory \\ of Nanobiological Technology of National \\ Health Commission, Xiangya Hospital, \\ Central South University, Changsha, Hunan, \\ 4I0008, People's Republic of China; \\ ${ }^{3}$ Department of Neurology, Xiangya \\ Hospital, Central South University, \\ Changsha, Hunan, 410008, People's Republic \\ of China; ${ }^{4}$ National Clinical Research Center \\ for Geriatric Disorders, Xiangya Hospital, \\ Central South University, Changsha, Hunan, \\ 410008, People's Republic of China; ${ }^{5}$ Clinical \\ Research Center for Cerebrovascular \\ Disease of Hunan Province, Central South \\ University, Changsha, Hunan, 410008, \\ People's Republic of China
}

*These authors contributed equally to this work

Correspondence: Xiangrui Yang; Jian Xia Email yangxr@csu.edu.cn;

xjian1216@csu.edu.cn
Introduction: Although the preparation of lipid nanoparticles (LNPs) achieves great success, their retention of highly hydrophobic drugs is still problematic.

Methods: Herein, we report a novel strategy for efficiently loading hydrophobic drugs to LNPs for stroke therapy. Oleoylethanolamide (OEA), an endogenous highly hydrophobic molecule with outstanding neuroprotective effect, was successfully loaded to OEASPC\&DSPE-PEG lipid nanoparticles (OSDP LNPs) with a drug loading of $15.9 \pm 1.2 \mathrm{wt}$ $\%$. Efficient retention in OSDP LNPs greatly improved the pharmaceutical property and enhanced the neuroprotective effect of OEA.

Results: Through the data of positron emission tomography (PET) and TTC-stained brain slices, it could be clearly visualized that the acute ischemic brain tissues were preserved as penumbral tissues and bounced back with reperfusion. The in vivo experiments stated that OSDP LNPs could significantly improve the survival rate, the behavioral score, the cerebral infarct volume, the edema degree, the spatial learning and memory ability of the MCAO (middle cerebral artery occlusion) rats.

Discussion: These results suggest that the OSDP LNPs have a great chance to develop hydrophobic OEA into a potential anti-stroke formulation.

Keywords: stroke, oleoylethanolamide, OEA, lipid nanoparticles, neuroprotective, positron emission tomography, PET

\section{Introduction}

The integration of drug and amphipathic molecules into nano-assembly achieves great success in optimizing the pharmaceutical properties of insoluble drugs. ${ }^{1-4}$ There are two primary procedures in an integration process: the crystallization of the drug and the self-assembly of the amphipathic molecules. ${ }^{5,6}$

When the drug is the chief component, its crystallization would be the main process. ${ }^{7,8}$ Serving as the crystallization inhibitor and stabilizer, the amphipathic molecules would co-precipitate on the drug crystals, terminating their growth and stabilizing them. ${ }^{9}$ As a result, micro- or nanocrystals would obtain instead of bulk crystals. For instance, 10-hydroxycamptothecin could assemble into nanofibers, which would grow into comet-shaped nanocrystalline assemblies under the guidance of a block copolymer additive- PLGA. ${ }^{5}$ The main advantage of the drug nanocrystals is the high drug loading, which might come up to more than $50 \% .{ }^{10,11}$ 
However, the high drug loading brings out the long-term instability and severe burst release. To address the problem, high amounts of amphipathic molecules are involved in nanodrugs. In this case, the self-assembly of the amphipathic molecules became the main process, accompanied by the co-precipitate of the drug into the assemblies. ${ }^{12}$ The LNPs, with the drug uniformly dispersed, were prepared via this strategy. ${ }^{13-16}$ Compared with the nanocrystals, the LNPs possessed significantly enhanced stability and controlled drug release properties. Until now, a number of LNPs have received regulatory approval, and many more are in the pipeline. ${ }^{17,18}$ The therapeutic benefits of the LNPs technology rely on the ability of long-circulation to increase the efficiency of chemotherapeutic agents by delivering high concentrations of the drug to tumor sites while reducing their accumulation in normal tissues to minimize the side-effect. ${ }^{19}$ Hence, the drug loading property of the LNPs is one of the key factors which influence their efficiency. However, the retention properties of drugs in LNPs are largely drugdependent. ${ }^{20}$ Loading the drug in response to transmembrane $\mathrm{pH}$ gradients would further increase the drug loading and stability. ${ }^{21}$ Via the technology, weak-base drugs, originally difficult to retain in LNPs, could receive a relatively high retention property. ${ }^{22,23}$ However, the retention of highly hydrophobic drugs in LNPs is still problematic. ${ }^{24-26}$ For instance, the loading efficiency of paclitaxel by different methods varies from $1.5 \%$ to $4 \%$ by weight. ${ }^{27,28}$ This extremely low drug loading is due to the highly hydrophobic property of paclitaxel. ${ }^{29}$ Current strategies to develop LNPs-based formulations of highly hydrophobic drugs have focused on the incorporation of these drugs in the lipid bilayer of LNPs. ${ }^{20}$ However, these formulations could not possess a high drug loading, and the drug rapidly exchanges from the liposome bilayer, leading to their instability in the blood circulation. ${ }^{24}$

Till now, stroke has been a leading cause of death worldwide. ${ }^{30}$ However, success in clinical stroke trials has remained elusive, mainly owing to the complex pathophysiology of stroke. ${ }^{31-34}$ Although it is well known that modulating the inflammatory process plays a paramount role in stroke-fighting, it is still a challenging clinical problem to comb out or even reduce the inflammation in stroke. ${ }^{35-38}$ Our team found that OEA could protect against ischemic injury via reducing inflammation. ${ }^{39-41}$ However, OEA is a water-insoluble molecule and could not be efficiently absorbed. To enhance the bioavailability of OEA, hydrogen-bond-based, OEA-loaded LNPs were developed. ${ }^{42}$ By forming hydrogen bonds between the drug and soybean lecithin (SPC), the drug could be efficiently and firmly loaded in LNPs, possessing a high drug loading and enhanced controlled release property. ${ }^{43-45}$ Based on this method, we report a novel strategy here, which could cleverly combine the self-assembly of the amphipathic molecules with the crystallization of the drug. With the crystallization of hydrophobic OEA inside the OSDP LNPs, the drug loading increased by four times compared to those of traditional methods. In the in vivo experiments, the OSDP LNPs possessed an outstanding neuroprotective effect and achieved enhanced therapeutic efficiency in the treatment of cerebral injury caused by MCAO.

\section{Materials and Methods \\ Materials}

All chemical reagents were of analytical grade and used without further purification unless otherwise stated. OEA (purity grade $>90.0 \%$ ) and SPC (soybean phosphatidylcholine, purity grade $>90.0 \%$ ) were obtained from Shanghai Macklin Biochemical Co., Ltd (Shanghai, China). DSPE-PEG (MWCO 2000) was purchased from Ponsure Biotechnology Co., Ltd (Shanghai, China). Dichloromethane (DCM) and tetrahydrofuran (THF) were provided by Sinopharm Chemical Reagent Co., Ltd. (Shanghai, China). 2,3,5-Triphenyltetrazolium chloride (TTC) was obtained from Shanghai Yuanye BioTechnology Co., Ltd (Shanghai, China)

\section{Preparation of OEA-SPC Complex (OEA- SPC)}

Three milligrams of OEA powder and $15 \mathrm{mg}$ of SPC were dissolved in a glass pressure vessel with $8 \mathrm{~mL}$ of THF, accompanied by vigorous agitation at $40^{\circ} \mathrm{C}$ for $8 \mathrm{~h}$. Then, THF was removed via vacuum rotary evaporation with a rotary evaporator (RE-5299; YUHUA, Gongyi, China).

\section{Preparation of OSDP LNPs}

The OSDP LNPs were synthesized by a nanoprecipitation technique. In brief, OEA ( $5 \mathrm{mg}$ ) and OEA-SPC (3 mg of OEA) were codissolved in $10 \mathrm{~mL}$ of DCM, and then the clear homogeneous solution was dropwise $(0.2 \mathrm{~mL} / \mathrm{min})$ introduced into distilled water $(40 \mathrm{~mL})$ containing DSPEPEG (20 mg) under magnetic stirring (200 rpm/min). After stirring for 1 hour, the system turned into a stable, white $\mathrm{O} / \mathrm{W}$ suspension. Subsequently, the dichloromethane was 
rapidly removed by rotary evaporation, producing a clear suspension. Then, the suspension was extruded through polycarbonate membranes $(0.22 \mu \mathrm{m}$ pore diameter $)$, and the OSDP LNPs were completely prepared. The OEA-SPC LNPs were prepared in the same way except no extra OEA was added in this procedure and DSPE-PEG was replaced by SPC.

\section{Characterization}

The OEA-SPC was analyzed using XRD (Phillips X'pert Pro Super) and $\mathrm{H}^{1} \mathrm{NMR}$ (AVANCE III $400 \mathrm{MHz}$ ). The bulk OEA power and SPC were used as control.

The OSDP LNPs were also analyzed with XRD to examine the form of OEA in OSDP LNPs. Morphology of the OSDP LNPs was examined by TEM (Tecnai G2 F20, USA) at $200 \mathrm{kV}$. The size and zeta-potential values were determined by a Malvern Zetasizer Nano-ZS machine (Malvern Instruments, UK). Three parallel measurements were carried out to determine the average values. The content of OEA in OEA-SPC LNPs was determined by LC-MS (LCMS ORBITRAP VELOS PRO ETD, USA). The content efficiency was calculated by Equation (1):

Drug loading content of OEA(DL, \%) $=($ weight of OEA in NPs $) /($ weight of NPs $) \times 100 \%$

$$
\begin{aligned}
& \text { Entrapment efficiency }(\mathrm{EE}, \%) \\
& =(\text { weight of OEA in NPs }) /(\text { weight of feeding OEA }) \\
& \quad \times 100 \%
\end{aligned}
$$

\section{In vitro Drug Release Study}

The in vitro drug release studies of OSDP LNPs were performed via the dialysis technique. The bulk OEA powers, OEA-SPC LNPs and OSDP LNPs were dispersed in PBS buffer solution $(10 \mathrm{~mL})$ and placed in dialysis bags (MWCO $3500 \mathrm{Da}$ ). Then, the dialysis bags were immersed in PBS (0.1 M, $150 \mathrm{~mL}, \mathrm{pH} 7.4)$ and continuously oscillated in a shaker incubator $\left(180 \mathrm{rpm}, 37^{\circ} \mathrm{C}\right)$. At predetermined time points $(1,2,4,8,18,30$, and $48 \mathrm{~h}), 2 \mathrm{~mL}$ of the release medium was completely withdrawn and subsequently replaced with the $2 \mathrm{~mL}$ of fresh PBS. To guarantee a sink condition, the amount of OEA should be less than $3.5 \mathrm{mg}$ according to its solubility. All samples were assayed by LC-MS.

\section{Drugs Administration}

The OSDP LNPs were dispersed in saline with ultrasonic breaking. OEA and Twain 80 was dissolved in saline under strong shaking. Drugs $(1.5 \mathrm{mg} / \mathrm{kg}$, iv) were administered once in rats at the time of redispersion, and daily for 14 consecutive days after ischemia.

\section{Animals}

The experimental protocols were approved by the Animal Care and Use Committee of Medical College of Central South University in compliance with the NIH Guide for the Care and Use of Laboratory Animals (NIH Publications No. 80-23). Male Sprague Dawley (SD) rats weighing 260$280 \mathrm{~g}$ were purchased from Beijing Vital River Experimental Animal Co. (Beijing, China) and housed under a 12/12 $\mathrm{h}$ dark/light cycle in specific pathogen-free (SPF) conditions. The animals were fasted without food deprivation for 12 $\mathrm{h}$ before the MCAO procedure was performed.

\section{Preparation of the Focal Cerebral Ischemia Model}

Focal cerebral ischemia was induced by MCAO in adult male Sprague Dawley (SD) rats, as previously described. In brief, rats were anesthetized with chloral hydrate (400 $\mathrm{mg} / \mathrm{kg}$, ip). The 4-0 silicon rubber-coated nylon monofilament was inserted into the right internal carotid artery (ICA) of the rats through the external carotid stump, and past the ECA/ICA bifurcation to occlude the origin of the middle cerebral artery (MCA) at the junction of the circle of Willis. The monofilament was kept in place for 90 min then withdrawn. Sham-operated rats were treated with an identical surgery except that the intraluminal filament was not inserted. Rats were excluded if the hemorrhage was found in the brain slices or at the base of the circle of Willis during postmortem examination.

\section{Positron Emission Tomography}

PET scans were performed at various time points postoperation ( $0 \mathrm{~h}, 1 \mathrm{~h}$, and $2 \mathrm{~h}$ ), and image reconstruction was performed using a nanoScan ${ }^{\circledR}$ PET/CT (Mediso, HUN). MCAO rats $(\mathrm{n}=6)$ were injected with $\left[{ }^{18} \mathrm{~F}\right]$-2-fluoro2-deoxy-D-glucose $\left(\left[{ }^{18} \mathrm{~F}\right] \mathrm{FDG}\right)$ via the tail vein and 20 min PET scans were performed. Since the best imaging time of FDG was about 45 min after administration, FDG was injected $45 \mathrm{~min}$ before each detection time. 


\section{Measurement of Infarct Volume}

After PET scans, the rats $(\mathrm{n}=6)$ were decapitated at 24 $h$ post-reperfusion, and the brains were removed rapidly and cut into six 2-mm thick coronal sections, which were then stained with standard $2 \% \mathrm{TTC}$ at $37^{\circ} \mathrm{C}$ for $10 \mathrm{~min}$ followed by overnight immersion in $10 \%$ formalin. Images of the stained brain sections were captured using a digital camera. The infarct area on each TTC-stained section was measured with Image Tool 2.0 software and calculated as the infarct area thickness $(2 \mathrm{~mm})$. The summed infarct volume of all brain sections was calculated as the total infarct volume. To determine the extent of ipsilateral edema, the percentage increase in the ischemic hemisphere volume was calculated by Equation (3):

Ipsilateral oedema degree( $\%)$

$$
=\frac{(\text { ipsilateral volume }- \text { contralateral volume })}{\text { contralateral volume }} \times 100 \%
$$

\section{Measurement of the Survival Rate}

The survival rate of the rats was recorded $(n=12$ rats for the sham group and the nanodrug group, and $n=24$ rats for the MCAO group and the OEA group).

\section{Evaluation of Neurological Deficit}

At $1 \mathrm{~d}, 3 \mathrm{~d}, 5 \mathrm{~d}, 7 \mathrm{~d}$, and $14 \mathrm{~d}$ after reperfusion, rats $(\mathrm{n}=10)$ were evaluated neurologically by a single examiner who was blinded to the animal groups using the Garcia method. Animals were given a score of 0 to 18 (higher scores indicate greater function).

\section{Morris Water Maze Task}

Morris water maze (MWM) task was performed from day 15 to day 20 after reperfusion. The protocol followed the previous report. Acquisition training consisted of 5-days conditioning with five trials per day from day 15 to 19 . For each trial, the rats ( $\mathrm{n}=8$ for each group) were placed in water and allowed to swim for a maximum of $120 \mathrm{~s}$. If the rats found the platform, they were allowed to remain on it for $15 \mathrm{~s}$. If they cannot find the platform within $120 \mathrm{~s}$, they would be guided to the platform. The escape latency of finding the platform was recorded. On day 20, the platform was removed and rats were given one 120-s retention probe test. We recorded the swimming traces of the rats by a video camera with a computer via an image analyzer. The number of times each animal crossed the position where the platform had been previously located was measured by the analyzer.

\section{Immunofluorescence Staining and Cell Counting}

Rats (21 d after reperfusion) were anesthetized with chloral hydrate and perfused transcardially with ice-cold saline followed by perfusion with $4 \%$ paraformaldehyde. The brains were extracted and dehydrated with a $10 \%, 20 \%$, $30 \%$ sucrose gradient and then coronal sectioned $(30 \mu \mathrm{m})$ using a vibrating microtome (Leica, Wetzlar, Germany). The sections were incubated in PBS $(0.5 \%$ Triton X-100 and $10 \%$ goat serum) for $1 \mathrm{~h}$ at room temperature, following by incubation with rabbit polyclonal, TUNEL (Elabscience, CHN), GFAP (CST, USA), or rabbit polyclonal Iba-1 (Abcam, UK) at $4{ }^{\circ} \mathrm{C}$ overnight. After several PBS rinses, sections were incubated with Alexa Fluor 594 donkey antirabbit IgG (Abcam, UK) or Alexa Fluor 488 donkey antirabbit IgG (Abcam, UK). The number of TUNEL, GFAP or Iba1-positive cells was analyzed by fluorescence confocal microscopy (EX61, Olympus, Tokyo, Japan). The positive cells were counted in three randomly chosen squares of identical size $(460 \times 460 \mu \mathrm{m})$.

\section{Statistical Analysis}

The statistical significance of treatment outcomes was assessed using one-way/two-way analysis of variance for the differences within treatments followed by Tukey's post hoc test (Prism 7 for Windows, GraphPad Software Inc., USA); $\mathrm{P}<0.05$ was considered statistically significant in all analyses $(95 \%$ confidence level $)$.

\section{Results and Discussion Preparation and characterization of OSDP LNPs}

A key feature of the research is the precursor-mediated, controlled drug crystallization inside the LNPs. In order to obtain nanosized crystals in the LNPs, the traditional nanoprecipitation technique was optimized. The hydrogen bond was formed between OEA and SPC via our previous method, ${ }^{43-45}$ which was confirmed by $\mathrm{H}^{1} \mathrm{NMR}$ and XRD. In the $\mathrm{H}^{1} \mathrm{NMR}$ spectra (Figure 1A), the peak at $7.76 \mathrm{ppm}$ (ascribed to the $-\mathrm{NH}-$ of OEA), the peak at $4.63 \mathrm{ppm}$ (ascribed to the -OH of OEA), and the peak at $3.47 \mathrm{ppm}$ (ascribed to phosphatidylcholine of SPC) appeared to be obviously weakened in the OEA-SPC complex, illustrating the formation of hydrogen bonds. The disappearance of the sharp peaks of bulk OEA in the XRD 



Figure I H'NMR spectra (A) and XRD patterns (B) of OEA, SPC, OEA-SPC and OSDP LNPs. (C) TEM images of OSDP LNPs. (D) lllustration of the formation and the structure of the OSDP LNPs.

pattern of OEA-SPC also confirmed that the hydrogen bonds might have formed between OEA and SPC molecules (Figure 1B).

Afterward, extra OEA and OEA-SPC were dissolved in dichloromethane, which were then dispersed in DI water containing DSPE-PEG under ultrasonic conditions. After stirring for 1 hour, the system turned to a stable, white $\mathrm{O} / \mathrm{W}$ suspension with the dispersion of the organic droplets in the continuous phase. At this stage, there was an arrangement of DSPE-PEG molecules with the hydrophobic groups of DSPE around the organic droplets and the PEG groups in the continuous phase. The droplets could be seen as precursors, which might proceed via non-classical crystallization routes. Lastly, with dichloromethane rapidly removed by rotary evaporation, the multistage crystallization process proceeded. Due to the evaporation of the organic phase, water molecules entered the droplets, resulting in the precipitation and arrangement of the SPC-OEA complex. Hence, phospholipid bilayers were successfully prepared. The bond of OEA to SPC molecules would serve many nucleation sites, leading to a number of OEA crystal nucleus. The 
rapid evaporation of the organic phase would induce a high supersaturation, under which the OEA crystal nucleus grew into OEA nanocrystals and rapidly reached the thermodynamically stable crystalline form. Since the phospholipid bilayers were flexible, their shapes might be changed by OEA nanocrystals. Followed by extrusion through polycarbonate membranes $(0.22 \mu \mathrm{m}$ pore diameter), irregularly non-spherical shaped LNPs, with drug crystal inside and phospholipid bilayers outside, were successfully prepared. The representative structure illustration of the OSDP LNPs is depicted in Figure 1D.

As illustrated in Figure 1C and Figure S1, the OSDP LNPs were around $100 \mathrm{~nm}$ with an irregularly nonspherical shape. It could be noticed that the black fields inside the LNPs appear darker than the fields forming the contour of the LNPs, which appear to be a paler shade of grey (Figure 1C-b and c). The inside dark field might owe to the existence of OEA crystals, whose molecules were arranged well-ordered and closely. Since the electrons could not easily pass through the closely packed crystals, the inside field of the LNPs possessed a deep color. On the contrary, the phospholipid bilayers were loosely arranged, leading to a higher electron transmittance and hence a light shade. The two different colored sections also illustrated the delicate hierarchical architectures of the OSDP LNPs.

$\mathrm{X}$-ray diffraction was also employed to detect the form of OSDP LNPs. As shown in Figure 1B, the sharp peaks of bulk OEA would disappear with the formation of hydrogen bonds in the OEA-SPC complex. Nevertheless, a majority of sharp peaks of OSDP LNPs belong to OEA, suggesting its high crystallinity. Although the OEA within OSDP LNPs shows the same polymorphic form as their bulk counterparts, the width of the peaks has reduced a lot, which might attribute to the nanoscaled crystal size of OEA within OSDP LNPs. Meanwhile, the coverage of lipid bilayer on the OEA nanocrystals led to the weakness of OEA.

\section{Stability Test and in vitro Drug Release Study}

The biggest advantage of the OSDP LNPs lies in the sustained drug release property with a high drug loading. The drug loading of OSDP LNPs could reach up to $15.9 \pm 1.2 \mathrm{wt}$ $\%$, while that of the OEA-SPC LNPs collected from the traditional method ranged from about $0.5 \mathrm{wt} \%$ to $3.0 \mathrm{wt} \%$. With the formation of hydrogen bonds, the drug loading increased to about $8.0 \mathrm{wt} \%$. When the nanoscaled OEA crystal appeared within the LNPs, the drug loading was doubled to $15.9 \pm 1.2 \mathrm{wt} \%$. Although the drug loading of many LNPs might come up to more than $50 \mathrm{wt} \%$, the retention of highly hydrophobic drugs is still a critical bottleneck. The drug loading of many LNPs was less than $10 \mathrm{wt} \%$, or even less. In this research, the formation of hydrogen bonds, plus the crystallization within the LNPs, greatly enhanced the retention of highly hydrophobic OEA. A well-established study showed that drug release could be controlled by the drug-loading content of the particles. High drug loading means severe burst release, while poor drug loading brings well-controlled drug release. ${ }^{46}$ As to OSDP LNPs (igure S2), higher drug loading also led to a little burst release within the first $8 \mathrm{~h}$, which might come from the exposed OEA crystals and the OEA on the surface of the LNPs. Compared to the OEA crystals without the coverage of lipid bilayer, this slight burst release could be neglected. Just like the OEA-SPC LNPs with a drug loading of $8.2 \mathrm{wt}$ $\%$, the OSDP LNPs exhibited a remarkably prolonged and controlled drug release in the next $40 \mathrm{~h}$, which could almost be seen as a zero-order release. That is to say, the enhanced drug loading did not reduce the controlled release property of the LNPs. This was owing to the unique construction of the LNPs. Unlike other drug crystals, the OEA crystals were located in the center of the OSDP LNPs, covered with lipid bilayers and bonding with the SPC molecules, which would largely limit the diffusion of the drug. Hence, the OSDP LNPs possessed a steady sustained release pattern throughout the release period. Moreover, the lipid bilayers, the PEG chains, the monodisperse (PDI $=0.176$ ), the high zeta potential $(-16.1 \mathrm{mV}, \underline{\text { Figure } \mathrm{S} 3})$, and the small particle size contributed to the stability of the OSDP LNPs. With minor size changes, the OSDP LNPs could preserve stability in PBS (pH 7.4, $0.1 \mathrm{M}, 37{ }^{\circ} \mathrm{C}$ ) for 48 hours (Figure S4). On the contrary, the OEA-SPC LNPs $(\mathrm{d}=213 \mathrm{~nm})$ without the coating of PEG chains would aggregate within 6 hours. And the lyophilized OSDP LNPs redissolved easier than OEA-SPC LNPs. The redissolved dispersion of lyophilized OSDP LNPs possessed a size of about $180 \mathrm{~nm}$ and was still applicable for intravenous injection within 1 month, while the OEA-SPC LNPs could not (Figure S5). The reason was the strong hydrophilia of PEG, which could stabilize the LNPs and make them more soluble in water.

\section{Assessment of the Neuroprotective Effect of the OSDP LNPs in vivo}

To assess the in vivo neuroprotective effects of the OSDP LNPs on ischemic cerebral injury, systematic experiments 
were performed on rats. According to our previous study, ${ }^{39,47}$ OEA could obviously reduce the cerebral infarct volume of the brains from the MCAO rats. Hence, it could be speculated that a lot of ischemic tissues were preserved to be ischemic penumbra with the administration of OEA, which could be salvaged by timely reperfusion. To visualize the ischemic penumbra and its recovery, positron emission tomography (PET) of $\left[{ }^{18} \mathrm{~F}\right]$ FDG was employed to assess the cerebral glucose metabolism. ${ }^{48,49}$ PET data were acquired for 20 minutes at $0 \mathrm{~h}, 1 \mathrm{~h}$, and $2 \mathrm{~h}$ after reperfusion. Since the inflammation-related high- $\left[{ }^{18} \mathrm{~F}\right]$ FDG uptake might appear around 3 d post-reperfusion, ${ }^{50}$ PET data were collected within 2 $\mathrm{h}$ post-reperfusion to eliminate the effect of inflammation. As shown in Figure 2A, the right cerebral hemisphere of all the operated rats exhibited significantly reduced glucose metabolism compared with the left cerebral hemisphere at $0 \mathrm{~h}$. The reason was that the blood supply of the right cerebral hemisphere was cut off and $\left[{ }^{18} \mathrm{~F}\right]$ FDG could not arrive. Then, with $1 \mathrm{~h}$ of reperfusion, the hypoperfused area began to recover in all the operated rats, and their uptake of $\left[{ }^{18} \mathrm{~F}\right] \mathrm{FDG}$ began to increase. Interestingly, about $41.7 \%$ of the right cerebral hemisphere from the rats administered with OSDP LNPs exhibited significantly elevated signals compared with the normal tissues (the blue dashed outline in Figure 2A). And the enhancement extended to $83.6 \%$ of the right cerebral hemisphere at 2 $h$ post-reperfusion. The PET intensity of these regions was about $63.24 \pm 2.43 \mathrm{kbp} / \mathrm{mL}$, which was much higher than that of the left cerebral hemisphere $(51.76 \pm 2.58 \mathrm{kbp} / \mathrm{mL})$. In these regions, the cutting-off blood flow and thereby glucose supply is compensated by an increase of glucose uptake and phosphorylation rate to maintain cellular energy consumption, ${ }^{51}$ which might be used for repairing the cell damage caused by hypoperfusion. The enhanced glucose metabolism also stated the good cell viability in the region, and the region was called "the ischemic penumbra" in the clinic. The ischemic penumbra would recover and functioned well afterward, which could be verified via the TTC-stained brain slices (Figure 2B). It could be observed that almost all the hypoperfused brain tissues, whose blood supply had been cut off for $90 \mathrm{~min}$, were not infarcted via the administration of the OSDP LNPs. The results indicated that the hypoperfused brain tissues with elevated PET signals after reperfusion were mostly likely to recover. On the contrary, the other MCAO rats did not exhibit enhanced glucose metabolism in the ischemic brain tissues. Although the brains were recovering with reperfusion, the low-level glucose metabolism stated their terrible cell viability at $1 \mathrm{~h}$ postreperfusion. What's worse, an obvious infarction core appeared at $2 \mathrm{~h}$ post-reperfusion (The red dashed outline in Figure 2A). While the other hypoperfused brain tissues possessed similar glucose metabolism to that of the left cerebral hemisphere, they had suffered from irreparable damage owing to the acute ischemia and might ultimately die. Many hypoperfused brain tissues with certain PET signals at $2 \mathrm{~h}$ post-reperfusion were infarcted as shown in the TTC-stained brain slices of $24 \mathrm{~h}$ post-reperfusion (Figure 2B). Then the cerebral infarct volume and the cerebral edema degree of all the rats were calculated (Figure 2C and D). With a cerebral infarct volume of about $372.2 \pm 26.9 \mathrm{~mm}^{3}$ and a cerebral edema degree of $13.6 \pm 1.0 \%$, the operated rats without treatment had severe brain damage, which would lead to a serious neurological deficit. With the treatment of free OEA, the cerebral infarct volume of the rats was reduced to 332.2 $\pm 35.3 \mathrm{~mm}^{3}$ and the cerebral edema degree to $11.3 \pm 1.1 \%$. However, there was no significant difference between the OEA and MCAO groups ( $\mathrm{P}>0.05)$, which was in accordance with our previous studies. ${ }^{42}$ Nevertheless, the cerebral infarct volume was decreased to $78.2 \pm 18.4 \mathrm{~mm}^{3}$, and the cerebral edema degree was also decreased to a slight level. The data stated that the rats treated with OSDP LNPs suffered from much less brain damage than the rats of the other groups. What's more, the photos of the TTC-stained brain slices, plus the PET data, forcefully demonstrated that the acute ischemic brain tissues could be preserved as penumbral tissues to a great extent via the administration of OSDP LNPs. And the preserved penumbral tissue would bounce back with reperfusion and possess an increased glucose metabolism within hours to compensate for the reduced supply, leading to a better and more rapid recovery.

As an acute disease, ischemic stroke possesses high mortality, and reducing death rates must be the primary task to a formulation for stroke. As shown in Figure 2E, only $45.8 \%$ of the MCAO rats could survive for $14 \mathrm{~d}$, and the administration of free OEA could not obviously change this data. In comparison, OSDP LNPs could effectively protect from MCAO and increase the survival rate to $83.3 \%$. Furthermore, we speculated that the rats administered with OSDP LNPs were more likely to die from the complications of MCAO, not from the cerebral injury. The small cerebral infarct volume illustrated that they suffered from a little cerebral injury (Figure S6). The 

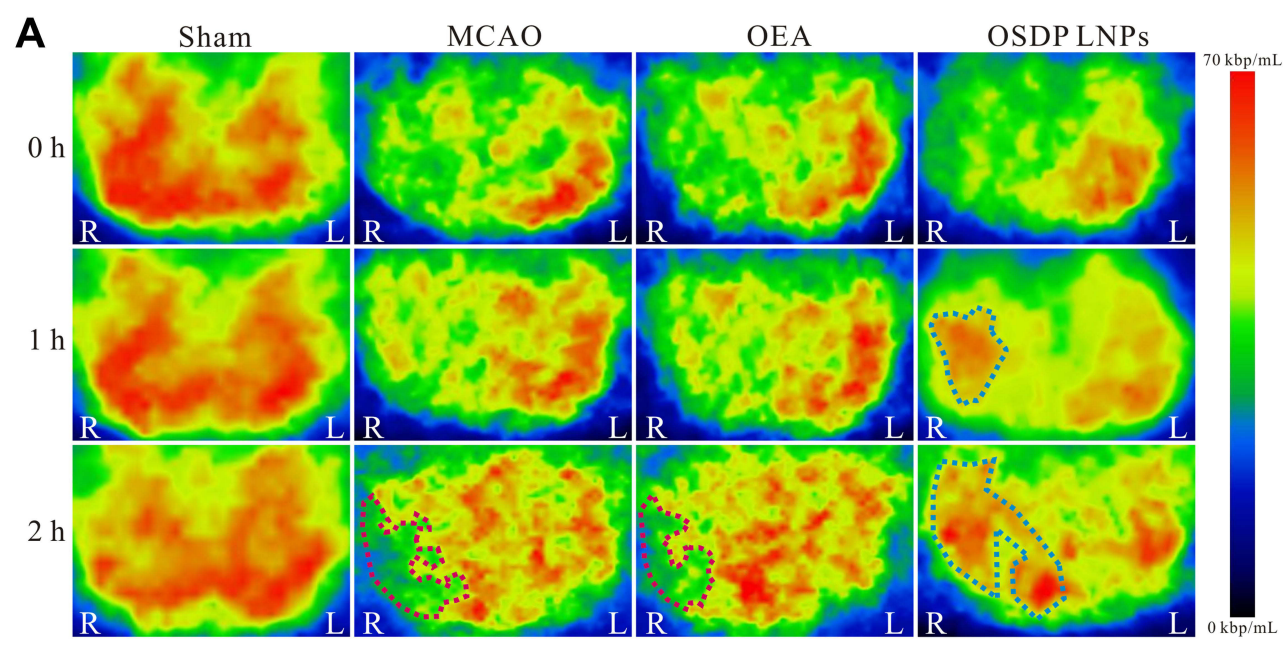

B
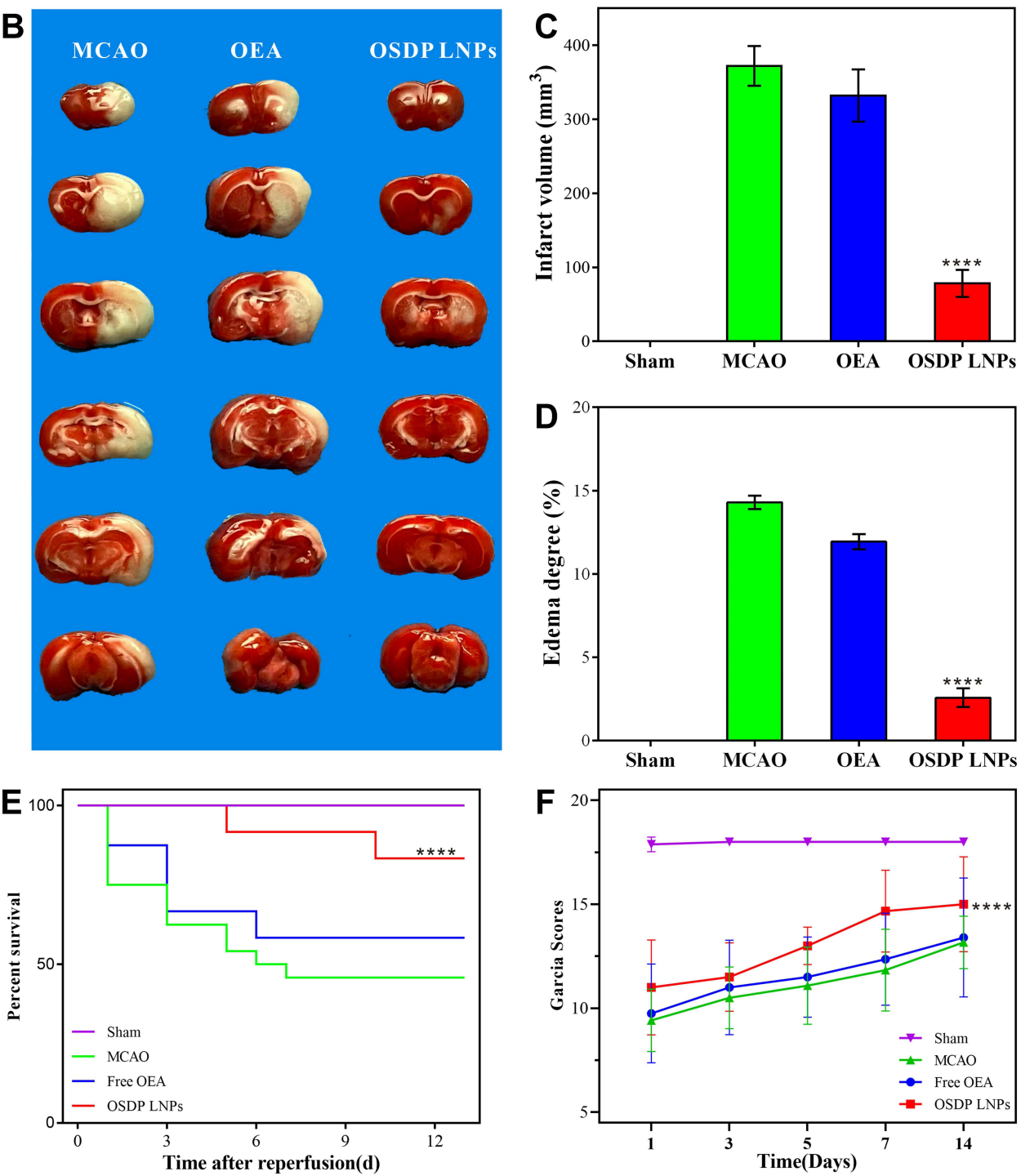

Figure 2 Therapeutic effects of the indicated formulations $(0.9 \% \mathrm{NaCl}$ (Sham), OEA and OSDP LNPs, ([OEA]=I.5 mg/kg) on cerebral damage after MCAO in rats. (A) PET images of $\left[{ }^{18} \mathrm{~F}\right] \mathrm{FDG}$ in rat brain at $0 \mathrm{~h}, \mathrm{I} \mathrm{h}$, and $2 \mathrm{~h}$ after MCAO. Red dashed outline represent the infarct core and the blue dashed outline represent the acute viable tissue. The infarct volume (B and $\mathbf{C})$, the edema degree $(\mathbf{D})$, the survival curves $(\mathbf{E})$, and the behavioral scores $(\mathbf{F})$ were detected. Data are expressed as mean $\pm S E M$. $n=24$ rats per group. Right middle cerebral artery was occluded. (****P<0.000I, compared to MCAO group.). 
result indicated that the administration of OSDP LNPs might extend the time window for beneficial reperfusion.

Since the behavior of the stroke patients was one of the most important evaluation indicators in the clinic, the behavior ability of the rats was evaluated via the Garcia method. The Garcia scores of the rats were evaluated at 1 , $3,5,7$, and $14 \mathrm{~d}$ after operation. As shown in Figure 2F, the sham-operated rats got full marks, while the operated rats only got about 10 points in the first assessment, illustrating the neurological function deficit of the models and the success of our operation. The rats administered with OSDP LNPs performed much better than those of the other two operated groups throughout the assessment $(\mathrm{p}<0.01)$. In addition to getting higher scores, the rats administered with OSDP LNPs maintained an enhanced recovery rate within the first week after operation, which might come from the preserved penumbral tissue. Under the protection of OSDP LNPs, more penumbral tissues were preserved and revived with reperfusion, leading to a better and more rapid recovery. On the contrary, OEA seemed to have no effect on improving neurological function deficit, owing to its extremely low bioavailability.

\section{Morris Water Maze Task}

Learning and memory are the most important computational strategies of the brain, which might be impaired by ischemia stroke. To examine the effects of OSDP LNPs on spatial learning and memory, rats were exposed to the water maze task. Between day 15 and 19 after operation, the rats were trained with five trials per day. Spatial learning ability was assessed by escape latency (the time required to find the platform in training). Compared with the sham group, the operated rats without treatment exerted much longer escape latency, illustrating their impaired spatial learning ability (Figure 3A). Owing to their severe brain damage, two rats even could not find the platform within 120 seconds in the third training, while all the sham rats could reach the platform within 60 seconds in the first training. Compared with those of the MCAO group, the rats treated with OSDP LNPs needed less time to find the platform, stating their improved spatial learning ability. Moreover, the training seemed to be more effective to the rats of the nanodrug group, leading to a result that the escape latency was decreased from $75 \mathrm{~s}$ in the first training to $23 \mathrm{~s}$ in the fourth training (Figure 3A). The result also illustrated their strong learning ability. At last, the data had no significant difference with that of the sham group, forcefully illustrating that the OSDP LNPs treatment effectively protected the spatial learning ability of the operated rats. Interestingly, the sham-operated rats exhibited significantly smarter than the operated rats after the first training. When entering the water maze in the second training, many of them looked around to confirm the position of the platform, and straightly swam to it. Two rats treated with OSDP LNPs began to have the same performance in the fourth training, while the rats of the other groups just swam around to find the platform throughout the training.

When the platform was removed, the rats of the sham group and the nanodrug group exhibited markedly increased target crossing times (Figure 3B), indicating their stronger potential memory for the removed platform. Moreover, it could be seen from the traces that the rats of the sham group and the nanodrug group always swam around the position of the removed platform (Figure 3D). This strongly indicated that they had a significant purpose and strong memory for the removed platform. On the contrary, the rats of the other groups possessed much less target crossing times and almost random traces, indicating their little memory for the platform. The results stated that the OSDP LNPs greatly ameliorated ischemia-induced spatial memory impairment.

\section{Immunofluorescence Staining and Cell Counting}

The intractable sequelae of stroke mostly resulted from the injury of neurons, which were really hard to recover. Here, TUNEL staining was employed to evaluate the apoptosis of the neurons in hippocampal CA1, which was mainly involved in memory and cognition. As shown in Figure 3C and $\mathrm{E}$, no apoptotic cell was detected in the hippocampal CA1 of the sham-operated rats, while a significant increase in apoptotic cells could be found in those of the MCAO group and the OEA group. The results stated that the MCAO was likely to induce the apoptosis of the neurons, and this could not be improved by the administration of free OEA. In contrast, only one apoptotic cell was observed in the field of the nanodrug group. This significant decrease was likely related to the improved learning and memory ability of the rats of the nanodrug group. The result also indicated the nice neuroprotective effect of OSDP LNPs.

According to our previous studies, ${ }^{39,47}$ OEA could inhibit the inflammation of reperfusion, which was one of the most primary causes of brain injury. Microglia and 




D

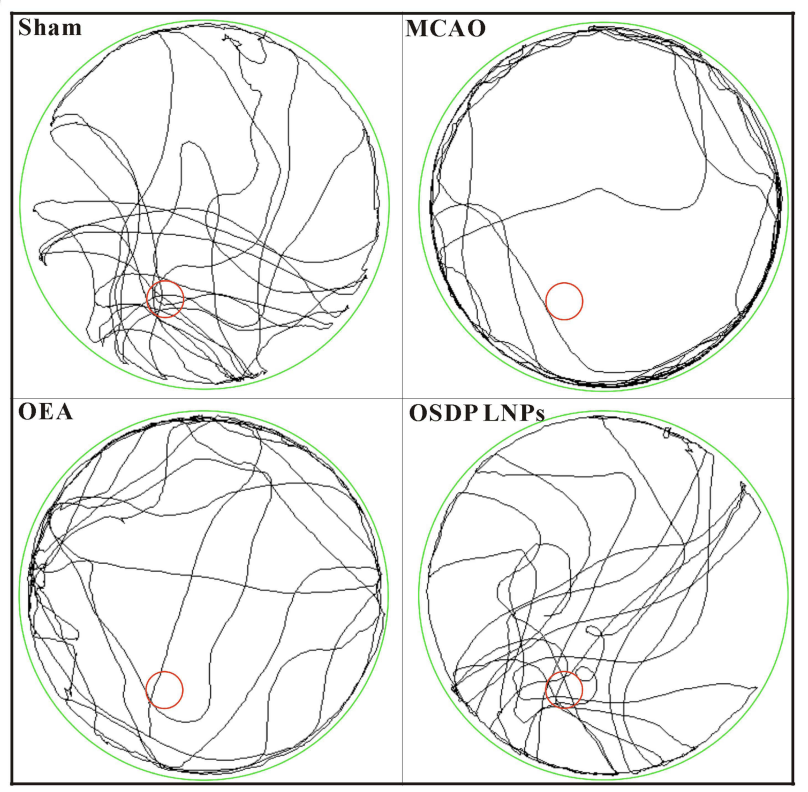

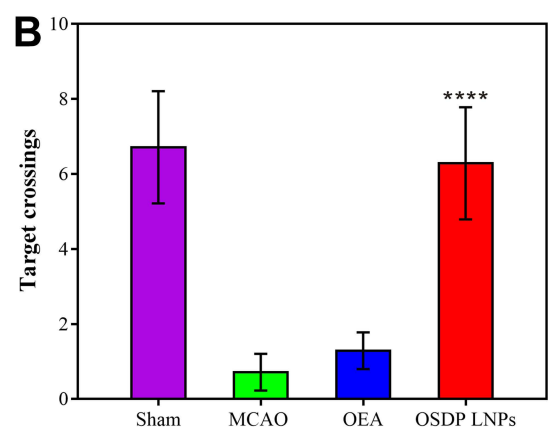

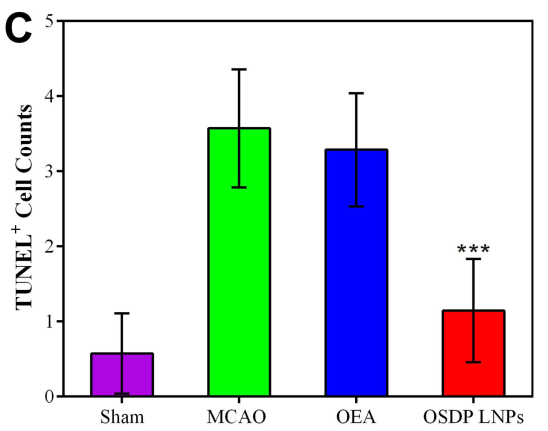

E

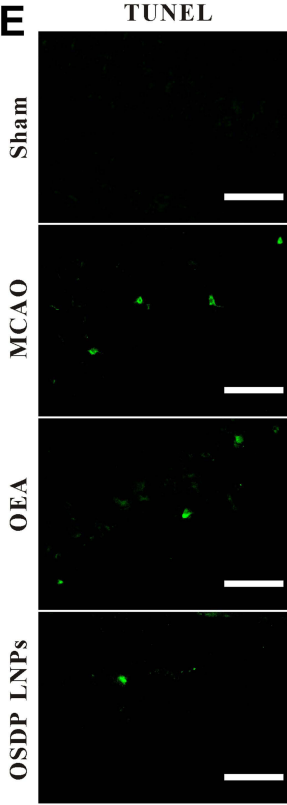

Merge

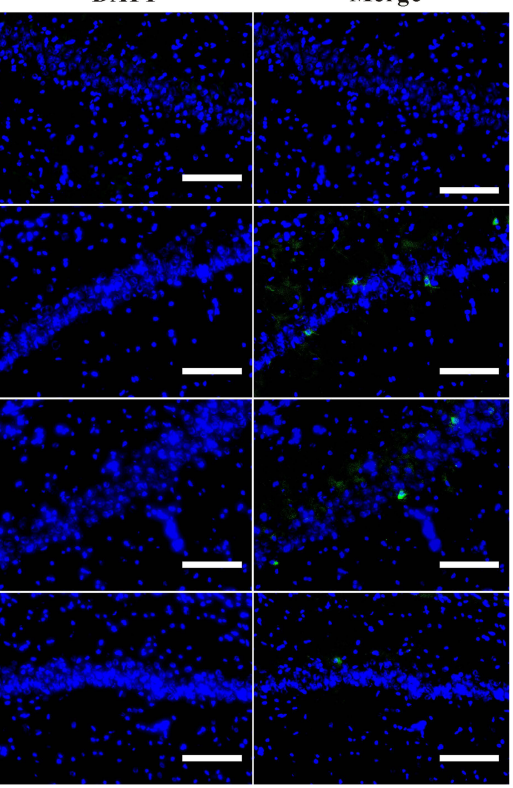

Figure 3 The effect of the formulations on MCAO-induced spatial cognitive deficits. The escape latency (A), the target crossings (B) and swimming traces (D) in the Morris water maze task were recorded. (C and E) Representative pictures of the hippocampal CAI stained with TUNEL (scale bars: $125 \mu \mathrm{m}$ ). Data are expressed as mean \pm SEM. $\mathrm{n}=10-12$ rats per group. $(* * \mathrm{P}<0.01, * * * \mathrm{P}<0.00 \mathrm{I}, * * * * \mathrm{P}<0.000 \mathrm{I}$, compared to MCAO group).

astrocyte play paramount roles in brain inflammation, which could be specifically marked by Iba- 1 and GFAP, respectively. Hence, the quantity of expressed Iba-1 and GFAP was used to evaluate the inflammation of reperfusion. As depicted in Figure 4, both markers suggested that the inflammation was slight in the sham-operated rats, while a significant increase could be observed in the cortex around the ischemic focus of the MCAO group and OEA group. With the administration of OSDP LNPs, the inflammation was decreased to a low level. The result indicated that the OSDP LNPs could greatly alleviate the inflammation induced by ischemic reperfusion and therefore provide significant neuroprotective effects.

\section{Conclusions}

In summary, the study demonstrates a simple but novel method to fabricate internal crystallized LNPs to efficiently encapsulate highly hydrophobic OEA. The hydrogen bond within OEA-SPC totally changed the form of OEA and enhanced its solubility. Then, the crystallization of OEA inside the OSDP LNPs further enhanced the drug loading; hence, the bioavailability of OEA would highly increase. With further studies focused on the assessment of the neuroprotective effect of the LNPs, the various indicators of the MCAO rats, including the survival rate, the behavioral score, the spatial learning and memory ability, the cerebral infarct volume, the edema degree, the apoptosis of the neurons and the inflammation within the brain, were greatly improved via the administration of OSDP LNPs. Furthermore, it could be clearly visualized that almost all the acute ischemic brain tissues were preserved under the protection of OSDP LNPs and quickly recovered with reperfusion. These results suggest that the internal crystallized LNPs have potential 

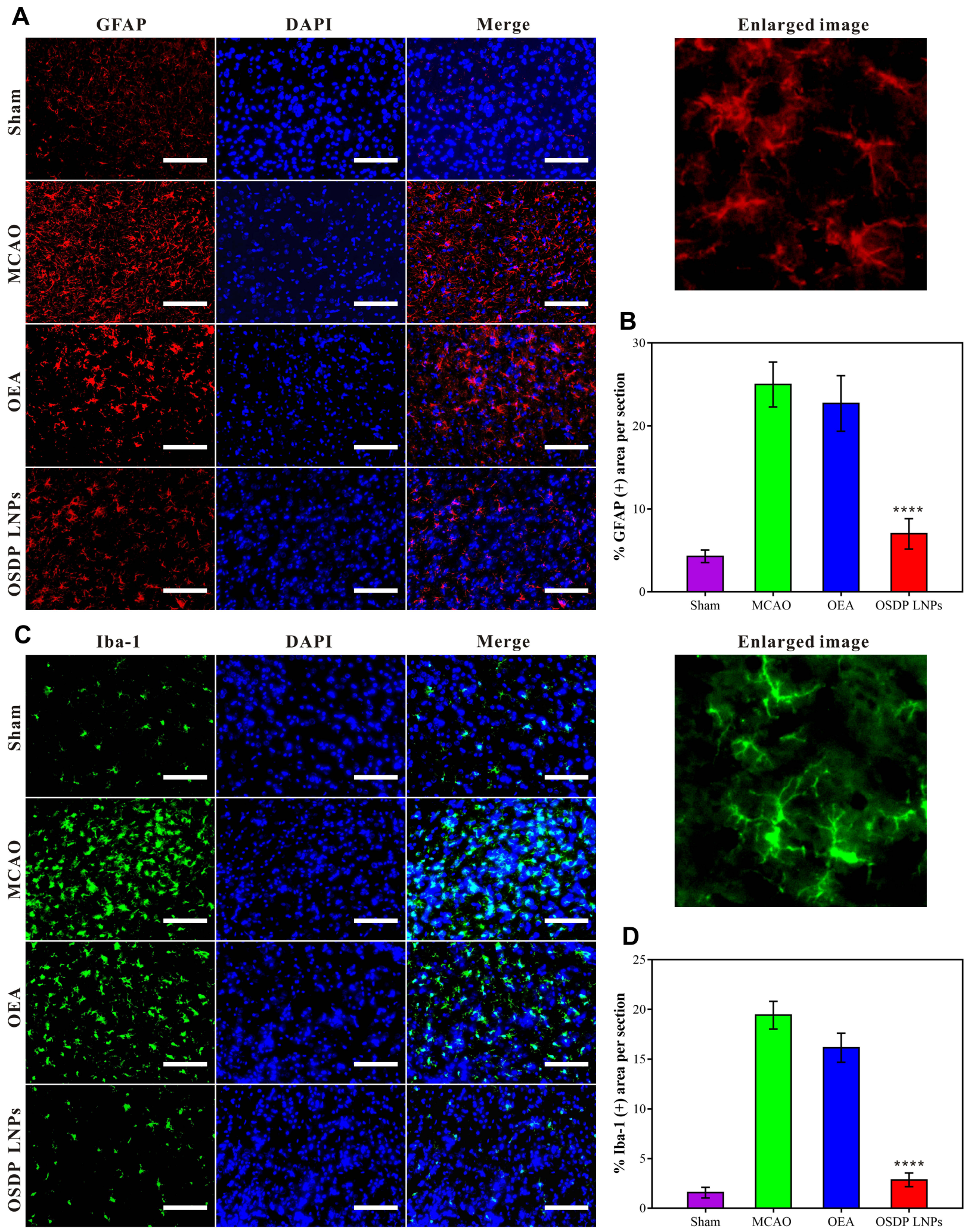

Figure 4 Quantification of the area of GFAP-positive stain (A and B) and the area of IBA-I-positive stain (C and D) from the cortex around the ischemic focus. Scale bars: $125 \mu \mathrm{m}$. (**** $\mathrm{P}<0.000 \mathrm{I}$, compared to MCAO group). 
applications in the field of hydrophobic drug delivery, and the OSDP LNPs may open new opportunities for the clinic use of hydrophobic OEA as a potential anti-stroke candidate.

\section{Abbreviations}

OEA, oleoylethanolamine; PET, positron emission tomography; MCAO, middle cerebral artery occlusion; OSDP LNPs, OEA-SPC\&DSPE-PEG LNPs; FDG, $\left[{ }^{18} \mathrm{~F}\right]-$ 2-fluoro-2-deoxy-D-glucose.

\section{Ethics Approval and Consent to Participate}

All procedures were carried out in accordance with the National Institutes of Health Guide for the Care and Use of Laboratory Animals and were approved by the Animal Ethical and Welfare Committee of Central South University.

\section{Acknowledgments}

This work was supported by National Natural Science Foundation of China (81901221 and 82001273) and Natural Science Foundation of Hunan province (S2021JJQNJJ1510).

\section{Disclosure}

The authors report no conflicts of interest in this work.

\section{References}

1. Yi X, Dai J, Han Y, et al. A high therapeutic efficacy of polymeric prodrug nano-assembly for a combination of photodynamic therapy and chemotherapy. Commun Biol. 2018;1:202. doi:10.1038/s42003018-0204-6

2. Shamay Y, Shah J, Isik M, et al. Quantitative self-assembly prediction yields targeted nanomedicines. Nat Mater. 2018;17(4):361. doi:10.1038/s41563-017-0007-z

3. Shi J, Kantoff PW, Wooster R, Farokhzad OC. Cancer nanomedicine: progress, challenges and opportunities. Nat Rev Cancer. 2017;17 (1):20-37. doi:10.1038/nrc.2016.108

4. Rosenblum D, Joshi N, Tao W, Karp JM, Peer D. Progress and challenges towards targeted delivery of cancer therapeutics. Nat Commun. 2018;9:1410. doi:10.1038/s41467-018-03705-y

5. Yang X, Wu S, Li Y, et al. Integration of an anti-tumor drug into nanocrystalline assemblies for sustained drug release. Chem Sci. 2015;6(3):1650-1654. doi:10.1039/C4SC03392B

6. Luo C, Sun B, Wang C, et al. Self-facilitated ROS-responsive nanoassembly of heterotypic dimer for synergistic chemo-photodynamic therapy. $J$ Controlled Release. 2019;302:79-89. doi:10.1016/j. jconrel.2019.04.001

7. Mann S. Self-assembly and transformation of hybrid nano-objects and nanostructures under equilibrium and non-equilibrium conditions. Nat Mater. 2009;8(10):781-792. doi:10.1038/nmat2496
8. Keck CM, Muller RH. Drug nanocrystals of poorly soluble drugs produced by high pressure homogenisation. Eur $J$ Pharm Biopharmaceutics. 2006;62(1):3-16. doi:10.1016/j.ejpb.2005.05.009

9. Li FY, Qin Y, Lee J, et al. Stimuli-responsive nano-assemblies for remotely controlled drug delivery. $J$ Controlled Release. 2020;322:566-592. doi:10.1016/j.jconrel.2020.03.051

10. Shen $\mathrm{S}, \mathrm{Wu}$ Y, Liu Y, Wu D. High drug-loading nanomedicines: progress, current status, and prospects. Int $J$ Nanomedicine. 2017;12:4085-4109. doi:10.2147/IJN.S132780

11. Jermain SV, Brough C, Williams RO. Amorphous solid dispersions and nanocrystal technologies for poorly water-soluble drug delivery An update. Int J Pharm. 2018;535(1-2):379-392. doi:10.1016/j. ijpharm.2017.10.051

12. Bodratti AM, Alexandridis P. Formulation of poloxamers for drug delivery. J Func Biomater. 2018;9(1):24.

13. Somasundar A, Ghosh S, Mohajerani F, et al. Positive and negative chemotaxis of enzyme-coated liposome motors. Nat Nanotechnol. 2019;14(12):1129. doi:10.1038/s41565-019-0578-8

14. Liu D, Chen B, Mo Y, et al. Redox-activated porphyrin-based liposome remote-loaded with indoleamine 2,3-dioxygenase (IDO) inhibitor for synergistic photoimmunotherapy through induction of immunogenic cell death and blockage of IDO pathway. Nano Lett. 2019;19(10):6964-6976. doi:10.1021/acs.nanolett.9b02306

15. Lakkadwala S, Rodrigues B, Sun C, Singh J. Dual functionalized liposomes for efficient co-delivery of anti-cancer chemotherapeutics for the treatment of glioblastoma. $J$ Controlled Release. 2019;307:247-260. doi:10.1016/j.jconrel.2019.06.033

16. Cheng MHY, Harmatys KM, Charron DM, Chen J, Zheng G. Stable J-aggregation of an aza-BODIPY-lipid in a liposome for optical cancer imaging. Angew Chem Int Edit. 2019;58(38):13394-13399. doi:10.1002/anie.201907754

17. Crommelin DJA, van Hoogevest P, Storm G. The role of liposomes in clinical nanomedicine development. $J$ Controlled Release. 2020;318:256-263. doi:10.1016/j.jconrel.2019.12.023

18. Bulbake U, Doppalapudi S, Kommineni N, Khan W. Liposomal formulations in clinical use: an updated review. Pharmaceutics. 2017;9(2):12. doi:10.3390/pharmaceutics 9020012

19. Kwon GS, Kataoka K. Block copolymer micelles as long-circulating drug vehicles. Adv Drug Deliv Rev. 2012;64:237-245. doi:10.1016/j. addr.2012.09.016

20. Allen TM, Cullis PR. Liposomal drug delivery systems: from concept to clinical applications. Adv Drug Deliv Rev. 2013;65(1):36-48. doi:10.1016/j.addr.2012.09.037

21. Drummond DC, Noble CO, Guo ZX, Hong K, Park JW, Kirpotin DB. Development of a highly active nanoliposomal irinotecan using a novel intraliposomal stabilization strategy. Cancer Res. 2006;66 (6):3271-3277. doi: 10.1158/0008-5472.CAN-05-4007

22. Tang W-L, Tang W-H, Szeitz A, Kulkarni J, Cullis P, Li S-D. Systemic study of solvent-assisted active loading of gambogic acid into liposomes and its formulation optimization for improved delivery. Biomaterials. 2018;166:13-26. doi:10.1016/j. biomaterials.2018.03.004

23. Liang J, Fang C, Wu W, Yu P, Gao J, Li J. Preparation and properties evaluation of a novel pH-sensitive liposomes based on imidazole-modified cholesterol derivatives. Int J Pharm. 2017;518 (1-2):213-219. doi:10.1016/j.ijpharm.2016.11.044

24. Yingchoncharoen P, Kalinowski DS, Richardson DR. Lipid-based drug delivery systems in cancer therapy: what is available and what is yet to come. Pharmacol Rev. 2016;68(3):701-787. doi:10.1124/ pr.115.012070

25. Liang X, Gao C, Cui L, Wang S, Wang J, Dai Z. Self-assembly of an amphiphilic janus camptothecin-floxuridine conjugate into liposome-like nanocapsules for more efficacious combination chemotherapy in cancer. Adv Mater. 2017;29(40):3135. doi:10.1002/ adma. 201703135 
26. Karim R, Palazzo C, Evrard B, Piel G. Nanocarriers for the treatment of glioblastoma multiforme: current state-of-the-art. $J$ Controlled Release. 2016;227:23-37. doi:10.1016/j.jconrel.2016.02.026

27. Yang T, Cui FD, Choi MK, et al. Enhanced solubility and stability of PEGylated liposomal paclitaxel: in vitro and in vivo evaluation. Int J Pharm. 2007;338(1-2):317-326. doi:10.1016/j.ijpharm.2007.02.011

28. Zhang JA, Anyarambhatla G, Ma L, et al. Development and characterization of a novel Cremophor (R) EL free liposome-based paclitaxel (LEP-ETU) formulation. Eur J Pharm Biopharmaceutics. 2005;59(1):177-187. doi:10.1016/j.ejpb.2004.06.009

29. Singla AK, Garg A, Aggarwal D. Paclitaxel and its formulations. Int J Pharm. 2002;235(1-2):179-192. doi:10.1016/S0378-5173(01) 00986-3

30. Virani SS, Alonso A, Benjamin EJ, et al. Heart Disease and Stroke Statistics - 2020 Update: a report From the American Heart Association. Circulation. 2020;141(9):e139-e596. doi:10.1161/ CIR.0000000000000757

31. Sommer CJ. Ischemic stroke: experimental models and reality. Acta Neuropathol. 2017;133(2):245-261. doi:10.1007/s00401-017-1667-0

32. Lee GA, Lin T-N, Chen C-Y, et al. Interleukin 15 blockade protects the brain from cerebral ischemia-reperfusion injury. Brain Behav Immun. 2018;73:562-570. doi:10.1016/j.bbi.2018.06.021

33. Chen C, Li T, Zhao Y, et al. Platelet glycoprotein receptor Ib blockade ameliorates experimental cerebral ischemia-reperfusion injury by strengthening the blood-brain barrier function and anti-thromboinflammatory property. Brain Behav Immun. 2018;69:255-263. doi:10.1016/j.bbi.2017.11.019

34. Ballarin B, Tymianski M. Discovery and development of NA-1 for the treatment of acute ischemic stroke. Acta Pharmacol Sin. 2018;39 (5):661-668. doi:10.1038/aps.2018.5

35. Stoll G, Nieswandt B. Thrombo-inflammation in acute ischaemic stroke - implications for treatment. Nat Rev Neurol. 2019;15 (8):473-481. doi:10.1038/s41582-019-0221-1

36. Rawlinson C, Jenkins S, Thei L, Dallas ML, Chen R. Post-ischaemic immunological response in the brain: targeting microglia in ischaemic stroke therapy. Brain Sci. 2020;10(3):159. doi:10.3390/ brainsci10030159

37. Peng X, Jing P, Chen J, Xu L. The role of circular RNA HECTD1 expression in disease risk, disease severity, inflammation, and recurrence of acute ischemic stroke. J Clin Lab Anal. 2019;33(7):e22954. doi: $10.1002 /$ jcla. 22954

38. Nakamura K, Shichita T. Cellular and molecular mechanisms of sterile inflammation in ischaemic stroke. J Biochem. 2019;165 (6):459-464. doi:10.1093/jb/mvz017

39. Zhou H, Yang W-S, Li Y, et al. Oleoylethanolamide attenuates apoptosis by inhibiting the TLR4/NF-kappa B and ERK1/2 signaling pathways in mice with acute ischemic stroke. Naunyn Schmiedebergs Arch Pharmacol. 2017;390(1):77-84. doi:10.1007/s00210-016-1309-4

40. Yang L, Guo H, Zhou H, et al. Chronic oleoylethanolamide treatment improves spatial cognitive deficits through enhancing hippocampal neurogenesis after transient focal cerebral ischemia. Biochem Pharmacol. 2015;94(4):270-281. doi:10.1016/j.bcp.2015.02.012
41. Zhou Y, Yang L, Ma A, et al. Orally administered oleoylethanolamide protects mice from focal cerebral ischemic injury by activating peroxisome proliferator-activated receptor alpha. Neuropharmacology. 2012;63(2):242-249. doi:10.1016/j.neuropharm.2012.03.008

42. Yang X, Xu L, Zhou J, et al. Integration of phospholipid-complex nanocarrier assembly with endogenous $\mathrm{N}$-oleoylethanolamine for efficient stroke therapy. $J$ Nanobiotechnology. 2019;17(1):8. doi:10.1186/s12951-019-0442-x

43. Li Y, Wu HJ, Jia MM, et al. Therapeutic effect of folate-targeted and PEGylated phytosomes loaded with a mitomycin C-soybean phosphatidylcholine complex. Mol Pharm. 2014;11(9):3017-3026. doi: $10.1021 / \mathrm{mp} 5001873$

44. Li Y, Lin J, Yang X, et al. Self-assembled nanoparticles based on amphiphilic anticancer drug-phospholipid complex for targeted drug delivery and intracellular dual-controlled release. ACS Appl Mater Interfaces. 2015;7(32):17573-17581. doi:10.1021/acsami.5b05038

45. Li Y, Wu HJ, Yang XR, et al. Mitomycin C-soybean phosphatidylcholine complex-loaded self-assembled PEG-lipid-PLA hybrid nanoparticles for targeted drug delivery and dual-controlled drug release. Mol Pharm. 2014;11(8):2915-2927. doi:10.1021/ mp500254j

46. Pang JM, Luan YX, Li FF, Cai XQ, Du JM, Li ZH. Ibuprofenloaded poly(lactic-co-glycolic acid) films for controlled drug release. Int $J$ Nanomedicine. 2011;6:659-665. doi:10.2147/IJN. S17011

47. Yang L, Guo H, Li Y, et al. Oleoylethanolamide exerts anti-inflammatory effects on LPS-induced THP-1 cells by enhancing PPAR alpha signaling and inhibiting the NF-kappa B and ERK1/2/AP-1/STAT3 pathways. Sci Rep. 2016;6:34611. doi:10.1038/srep34611

48. Kelly PJ, Camps-Renom P, Giannotti N, et al. Carotid plaque inflammation imaged by F-18-fluorodeoxyglucose positron emission tomography and risk of early recurrent stroke. Stroke. 2019;50:1766-1773. doi:10.1161/STROKEAHA.119.025422

49. Deng L, Wan H, Zhou H, Yu L, He Y. Protective effect of hydroxysafflor yellow A alone or in combination with acetylglutamine on cerebral ischemia reperfusion injury in rat: a PET study using F-18fuorodeoxyglucose. Eur $J$ Pharmacol. 2018;825:119-132. doi:10.1016/j.ejphar.2018.02.011

50. Fukumoto D, Hosoya T, Nishiyama S, et al. Multiparametric assessment of acute and subacute ischemic neuronal damage: a small animal positron emission tomography study with rat photochemically induced thrombosis model. Synapse. 2011;65:207-214. doi:10.1002/syn.20836

51. Walberer M, Backes H, Rueger MA, et al. Potential of early F-182-fluoro-2-deoxy-D-glucose positron emission tomography for identifying hypoperfusion and predicting fate of tissue in a rat embolic stroke model. Stroke. 2012;43:193-U359. doi:10.1161/ STROKEAHA.111.624551
International Journal of Nanomedicine

\section{Publish your work in this journal}

The International Journal of Nanomedicine is an international, peerreviewed journal focusing on the application of nanotechnology in diagnostics, therapeutics, and drug delivery systems throughout the biomedical field. This journal is indexed on PubMed Central, MedLine, CAS, SciSearch ${ }^{\mathbb{R}}$, Current Contents ${ }^{\mathbb{B}} /$ Clinical Medicine,
Journal Citation Reports/Science Edition, EMBase, Scopus and the Elsevier Bibliographic databases. The manuscript management system is completely online and includes a very quick and fair peer-review system, which is all easy to use. Visit http://www.dovepress.com/ testimonials.php to read real quotes from published authors. 